\title{
INVERSÃO UNIDIMENSIONAL DE PERFIS GEOFÍSICOS DE INDUÇÃO NA CARACTERIZAÇÃO DOS TURBIDITOS DA BACIA DE ALMADA - BA
}

\author{
Alfredo M. V. Carrasco, Kledson Tomaso, ${ }^{2}$ Antonio Abel González Carrasquilla ${ }^{3}$ \\ Recebido em 12 dez. 2003 / Aceito em 24 maio 200 \\ Received dec. 12, 2003/ Accepted may 24, 2004
}

\begin{abstract}
In this work, we developed an one-dimensional inverse algorithm, based in the dumped least square technique, to interpret electromagnetic induction geophysical well log data. The goal was the characterization of a turbidite geological system. Thus, the forward problem consists in the exact mathematical solution of the electromagnetic response of a stratified media crossed by a borehole. The inversion scheme was used to interpret apparent resistivity data obtained in a borehole drilled in a turbidity outcrop of Almada Basin, South Bahia State/Brazil. To validate our interpretation, geologists described the lithology from samples obtained and also using gamma ray log. With the developed algorithm, it was possible to describe the resistivity and thickness of the main layers along the well, besides the bias between final model and experimental data and the fitting error. Although we know that one-dimensional inversion is very unstable, it has some advantages, as a rapidly convergence when initial model is close the correct model.
\end{abstract}

Keywords: One-dimensional inversion, induction well logging, turbidites, Almada Basin

\section{RESUMO}

Neste trabalho, foi desenvolvido um algoritmo de inversão unidimensional baseado na técnica dos mínimos quadrados amortecidos, para interpretar dados geofísicos de perfis de indução. 0 objetivo consistiu na caracterização de um sistema geológico de turbiditos, utilizando um esquema de inversão, para interpretar os dados de resistividade aparente obtidos na perfilagem de poço na Bacia de Almada, no Sul do Estado da Bahia. 0 problema direto, por outro lado, consiste na solução matemática exata da resposta eletromagnética de um meio estratificado atravessado por um poço. Para validar a interpretação, foi utilizada a descrição geológica de testemunhos e amostras de calha conjuntamente com informação de perfis de raios gama. Desta forma, foi possível descrever os valores de resistividades e espessuras para cada camada ao longo do poço, assim como o desvio entre os dados experimentais e os do modelo final e 0 erro de ajuste.

Palavras-chave: Almada, inversão, perfil de indução, turbiditos.

\footnotetext{
1 Universidade Estadual do Norte Fluminense (UENF) - Laboratório de Engenharia e Exploração de Petróleo (LENEP) - Rodovia Amaral Peixoto, Km 163 - Avenida Brenand S/N - CEP: $27925-031$ - Imboassica - Macaé, RJ - Brasil - Telefone: +55 (22) 2773-6565 - Fax +55 (22) 2773-6564 - e-mail: alfredo@lenep.uenf.br

2 Universidade Estadual do Norte Fluminense (UENF) - Laboratório de Engenharia e Exploração de Petróleo (LENEP) - Rodovia Amaral Peixoto, Km 163 - Avenida Brenand S/N - CEP: 27925-031 - Imboassica - Macaé, RJ - Brasil - Telefone: + 55 (22) 2773-6565 - Fax + 55 (22) 2773-6564 - e-mail: tomaso@lenep.uenf.br

3 Universidade Estadual do Norte Fluminense (UENF) - Laboratório de Engenharia e Exploração de Petróleo (LENEP) - Rodovia Amaral Peixoto, Km 163 - Avenida Brenand S/N - CEP: $27925-031$ - Imboassica - Macaé, RJ - Brasil - Telefone: +55 (22) 2773-6565 - Fax +55 (22) 2773-6564 - e-mail: abel@lenep.uenf.br
} 


\section{INTRODUÇÃO}

Neste artigo, uma metodologia de inversão unidimensional (1D) foi utilizada para interpretar perfis resistivos de indução ILD (Induction Log Deep). Este tipo de interpretação tem sido muito usado para calcular a resposta de modelos de camadas plano-paralelas em métodos geofísicos como eletro-resistividade, polarização induzida e magnetotelúrico (WU, 1968). 0 algoritmo inverso baseia-se na técnica dos mínimos quadrados amortecidos, também conhecida como ridge-regression, método que foi proposto por Marquardt (1970). Assim, o objetivo foi auxiliar no zoneamento e na caracterização resistiva do sistema turbidítico da Bacia de Almada, no Sul do Estado da Bahia.

0 perfil estudado no poço SA-01 atravessa a Formação Urucutuca e está localizado à margem da estrada Ilhéus - Urucutuca, no Distrito de Sambaituba. 0 afloramento estudado apresenta, sob o ponto de vista geofísico, camadas finas plano-paralelas próprias dos turbiditos, que podem ser observadas na análise dos perfis de poço (TOMASO, 2003).

Muitas vantagens são consideradas quando se utiliza esse método de inversão: convergência a partir de modelos iniciais com pouca precisão, estabilidade na presença de ruído geológico e obtenção rápida de estimativas de parâmetros estatísticos (PELTON; RIJO; SWIFT, 1978). Desafortunadamente cada regressão requer muitas avaliações do problema direto, e para atingir velocidade a um custo razoável, é importan- te reduzir o tempo, otimizando o cálculo do problema direto. Isto foi realizado num trabalho anterior de Carrasco e Carrasquilla (2003).

Como na maioria dos métodos geofísicos, a unicidade da inversão não está garantida, mas quando os parâmetros iniciais estão próximos dos valores verdadeiros, a convergência fica garantida. Portanto, outro objetivo deste trabalho foi investigar a unicidade nos modelos geológicos empregados, e avaliar o erro resultante, empregando esse método de inversão.

\section{CONTEXTO GEOLÓGICO}

A Bacia de Almada, uma das várias bacias da margem continental brasileira, situa-se na porção sul do Estado da Bahia, entre os paralelos $14^{\circ} 15^{\prime}$ e $14^{\circ} 55^{\prime} \mathrm{S}$, limitada ao norte pelo alto de Itacaré que a separa da Bacia de Camamu, e ao sul pelo Alto de Olivença, que a separa da Bacia do Jequitinhonha. Inclui uma pequena porção emersa, com extensão de $200 \mathrm{~km}^{2}$ e espessura máxima de sedimentos de $1.800 \mathrm{~m}$ (Figura 1). Na plataforma continental, sua área atinge uma extensão maior, com cerca de $1.300 \mathrm{~km}^{2}$ e coluna sedimentar com $6.000 \mathrm{~m}$ de espessura (BRUHN; MORAES, 1989).

Os afloramentos da Bacia de Almada ocorrem sob a forma de um canyon semi-exumado, encravado no embasamento. Estes afloramentos foram bem estudados por Bruhn e Moraes (1989) e Men-

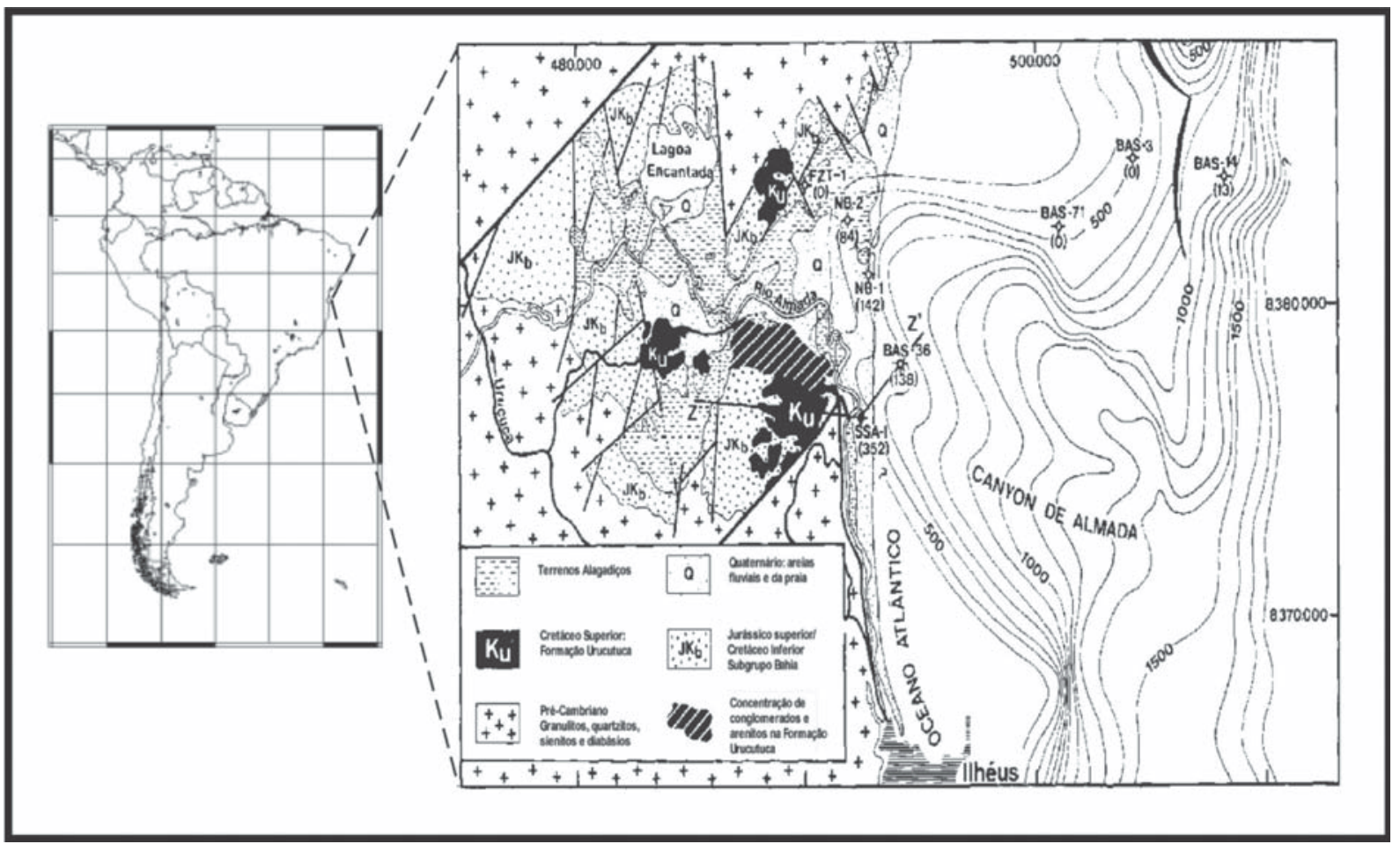

Figura 1 - Localização da área estudada. A geologia é mostrada no painel a esquerda. Modificada de (BRUHN; MORAES, 1989). Figure 1 - Area studied. Geology is shown on the left side. Modified of (BRUHM; MORAES, 1989) 
des (1998), sendo os únicos exemplos aflorantes dos turbiditos da megaseqüência marinha transgressiva da margem brasileira. Esses turbiditos possuem a mesma idade dos principais reservatórios de petróleo do Brasil, presentes nas Bacias de Campos, Espírito Santo e Sergipe/ Alagoas. Portanto, é importante caracterizar esse tipo de formação geológica da forma como é feito nosso estudo.

0 perfil estudado foi obtido no poço SA-01, com 255m de profundidade e 8,5 polegadas de diâmetro, localizado nas coordenadas $14^{\circ} 39^{\prime} 31^{\prime \prime}$ S e $39^{\circ} 06^{\prime} 05^{\prime \prime W}$. Os turbiditos presentes nesse poço são constituídos por formações geológicas areno-conglomeráticas e folhelhos de idades campaniana/maestrichtiana, que definem litoestratigraficamente a Formação Urucưtuca. № afloramento em questão foram identificados depósitos arenosos com estruturas geológicas do tipo wavy, linsen, ripples e mud coupplets. Existem também bioturbações por Ophiomorfa e geometria em cunhas largas que preenchem inclusões em onlap (D’AVILA, 2003).

\section{METODOLOGIA}

\section{Perfilagem de poço}

A perfilagem geofísica desempenha um papel fundamental na exploração de hidrocarbonetos, principalmente na avaliação das características petrofisicas dos reservatórios. Ela é um método que representa um pequeno percentual do custo total do poço, mas fornece importantes informações das propriedades das rochas presentes.

Na Bacia de Almada, os turbiditos estão aflorando, facilitando 0 seu estudo geológico de maneira direta. Esse fato não diminui a importância de um estudo através da perfilagem de poços, pois os seus resultados são obtidos nos poços em condições de rochas 'frescas' não intemperizadas, sem os eventuais processos geológicos que provocam a chegada desses pacotes turbiditicos na superfície, alterando sua geometria, assim como as suas propriedades físicas e geológicas.

Para desenvolver o nosso estudo utilizamos o perfil de indução 'Induction Log Deep' (ILD) no poço SA-01, o qual mede a condutividade elétrica (inverso da resistividade) das formações geológicas atravessadas pelo poço. Os perfis de resistividade podem ser utilizados para correlações geológicas e na determinação da resistividade verdadeira das rochas quando as camadas são bastante espessas. 0 principal problema encontrado nestes perfis está relacionado diretamente com a diferença existente entre os diâmetros das sondas e dos poços. Um significativo volume de lama influencia, predominantemente, a leitura final da resistividade afastando-a cada vez mais dos valores reais necessários para a realização dos cálculos petrofísicos quantitativos (NERY; FEITOSA; MANOEL FILHO, 1997). Neste trabalho, esses efeitos não foram corrigidos.

\section{0 problema direto}

0 desenvolvimento do algoritmo direto $1 \mathrm{D}$ está baseado na solução exała do problema de Green para a determinação do campo eletromagnético no interior de um meio estratificado, atravessado por um poço e excitado por uma ferramenta de poço composta por um transmissor e um receptor.

Para testar 0 algoritmo desenvolvido, consideramos um modelo formado por uma sequiência de 27 camadas, conhecido nos Estados Unidos como Formação Oklahoma, largamente utilizado como referencial na perfilagem de poços da indústria de petróleo. A Figura 2 mostra as resistividades aparentes calculadas para as sondas ILD, 2 C40 e 6FF40, todas da SCHLUMBERGER (CARRASCO; CARRASQUILLA, 2003).

\section{O PROBLEMA INVERSO}

Utilizando uma expansão em séries de Taylor, uma equação nãolinear do tipo:

$$
\Delta \mathrm{G}=\mathrm{A} \Delta \mathrm{P},
$$

pode ser linearizada na forma:

$$
\begin{aligned}
& \Delta \mathrm{G}_{\mathrm{i}}=\mathrm{G}\left(\mathrm{P}, \mathrm{X}^{\mathrm{i}}\right)-\mathrm{G}\left(\mathrm{P}^{0}, \mathrm{X}^{\mathrm{i}}\right), \mathrm{i}=1, \mathrm{~N} ; \\
& {[A]_{i j}=\left.\frac{\partial G(P, X)}{\partial P_{j}}\right|_{\substack{X=X^{I} \\
p=p^{0}}} ; \Delta \mathrm{P}_{\mathrm{j}}=\mathrm{P}_{\mathrm{j}}-\mathrm{P}_{\mathrm{j}}^{0} .}
\end{aligned}
$$

0 termo $\Delta \mathrm{G}$ representa a diferença entre a resistividade aparente medida no perfil de poço e a resistividade calculada pelo modelo inicial $\mathrm{P}^{0}$. 0 elemento do sistema matricial $\mathrm{A}_{\mathrm{ij}}$ é a derivada da Equação (1) com relação a i j-ésimo parâmetro do modelo avaliado com $\mathrm{P}^{0}$ no í-esimo espaçamento das camadas. 0 vetor $\Delta \mathrm{P}$ é a diferença entre os parâmetros do ajuste final e os do modelo inicial.

Assim, o procedimento de inversão consiste em encontrar um vetor de parâmetros que minimize $\Delta \mathrm{G}$, pois a Equação (1) foi estabelecida para linearizar 0 sistema, sendo a solução atingida após várias iterações. Este procedimento é repetido até que a diferença vetorial ' $G$ ' seja minimizada com base no critério dos mínimos quadrados.

A solução é dependente do modelo inicial. Assim, é importante utilizar no começo do processo de inversão, informação geológica adicional confiável para descrever o modelo inicial (INMAN et al., 1973). 


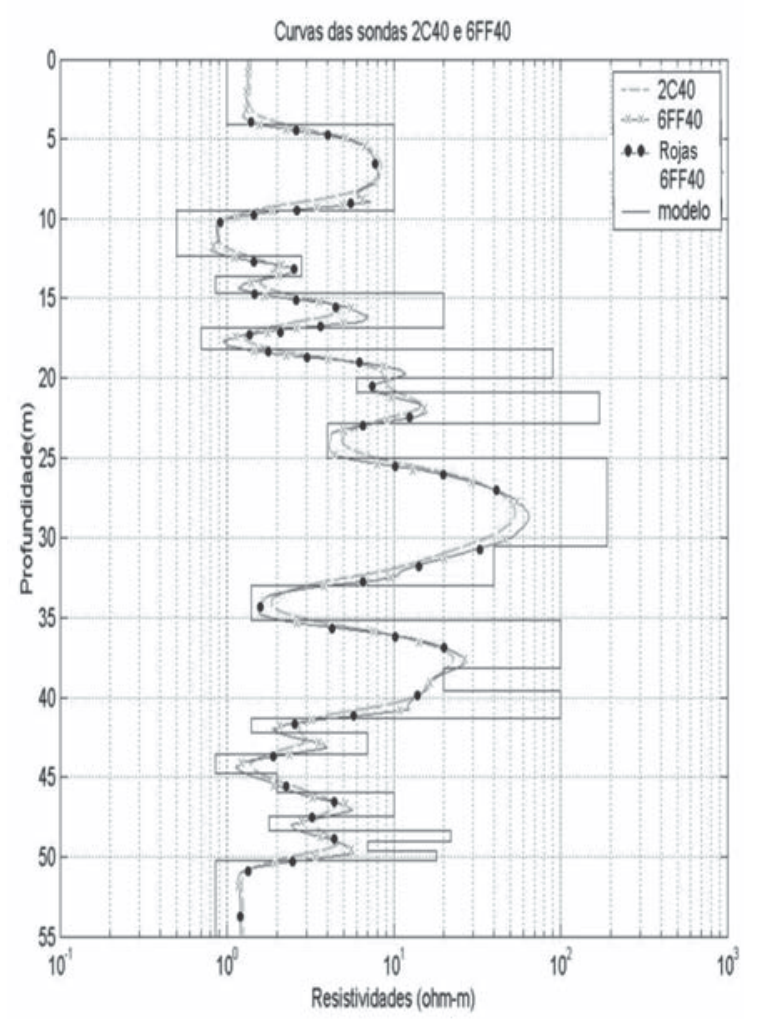

Figura 2 - Resultados do modelo 10 de Oklahoma Figure 2 - 10 model results from Oklahoma

\section{Mínimos quadrados simples}

Qualquer função matemática pode ser linearizada, como foi descrito na Equação (1), mas sempre existirá um erro $\xi$ nesse processo, na forma:

$\Delta \mathrm{G}=\mathrm{A} \Delta \mathrm{P}+\xi$,

sendo $\mathrm{A}$ a matriz sensibilidade que relaciona as variações nos parâmetros $\Delta \mathrm{P}$ às variações nos dados $\Delta \mathrm{G}$. Quando o valor de x é mínimo, podemos obter DP no sentido dos mínimos quadrados:

$\Delta \mathrm{P}=\left(\mathrm{A}^{\mathrm{T}} \mathrm{A}\right)^{-1} \mathrm{~A}^{\mathrm{T}} \Delta \mathrm{G}$

Nesta expressão simplesmente foi multiplicado, em ambos os lados da Equaçã̃o (1), o operador de inversão generalizado para sistemas determinados e sobredeterminados (PELTON; RIJ0; SWIFT, 1978):

$\mathrm{H}=\left(\mathrm{A}^{\mathrm{T}} \mathrm{A}\right)^{-1} \mathrm{~A}^{\mathrm{T}}$.
Para esses casos, a matriz A pode ser invertida rapidamente. Por tal razão, se multiplica ambos os lados da Equação (1) por A', onde ' $\mathrm{T}$ ' representa a matriz transporta. Desta forma:

$\mathrm{A}^{\mathrm{T}} \Delta \mathrm{G}=\mathrm{A}^{\mathrm{T}} \mathrm{A} \Delta \mathrm{P}$.

Se o problema fosse "bem posto" e o modelo inicial muito próximo da resposta final, poderíamos simplesmente inverter $\mathrm{A}^{\top} \mathrm{A}$ para obter $\Delta \mathrm{P}$. No entanto, é difíicil propor um modelo inicial preciso, e, freqüentemente, incluir parâmetros que não são exatos. Como resultado, A'A é aproximadamente singular e, pela Equação (3), pode-se produzir uma mudança nos parâmetros e incrementar os residuais dos números quadrados $\Delta \mathrm{G}^{\mathrm{\top}} \Delta \mathrm{G}$ (INMAN, 1975).

Uma vez que o modelo inicial é selecionado, o objetivo da inversão é minimizar 0 erro entre os dados calculados e os observados:

$\xi=\Delta \mathrm{G}^{\mathrm{T}} \Delta \mathrm{G}$

Se o problema fosse exatamente linear, somente uma determinação seria necessária. Entretanto, a maior parte dos problemas geofísicos são não-lineares e usualmente requerem muitas iterações para obter uma solução satisfatória a partir de um modelo inicial (PETRICK; PELTON; WARD, 1977).

\section{Mínimos quadrados amortecidos}

Para estabilizar $\mathrm{A}^{\mathrm{T}} \mathrm{A}$ e evitar divergência, Levenberg, Foster e Marquardt (Inman, 1975) propuseram a adição de uma pequena constante positiva ' $\mathrm{k}$ ' aos elementos da diagonal principal antes do processo de inversão, de tal maneira que a Equação (3) torna-se:

$\Delta \mathrm{P}^{*}=\left(\mathrm{A}^{\mathrm{T}} \mathrm{A}+\mathrm{k}\right)^{-1} \mathrm{~A}^{\mathrm{T}} \Delta \mathrm{G}$,

onde I é a matriz identidade. Num problema linear, um valor ótimo de " $k$ " é aquele que proporciona o melhor ajuste no sentido dos mínimos quadrados. Se o valor de "k" for muito alto, a Equação (7) se aproxima do método inverso do gradiente, que embora estável, converge lentamente. Se 'k' tiver valor próximo de zero, a Equação (7) se aproxima do método Newton-Raphson, que ao contrário do caso anterior, é rápido mas diverge. Na prática é recomendável testar vários valores de ' $\mathrm{k}$ ' numa escala logarítmica para minimizar o resíduo dos mínimos quadrados amortecidos. Essa técnica que altera 0 valor de ' $\mathrm{k}$ ' através de métodos iterativos é conhecida como método dos mínimos quadrados amortecidos ou 'ridge-regression' (PELTON; PELTON; WARD, 1978). 
A função do parâmetro de regularização ' $\mathrm{k}$ ' é a de amortecer pequenos autovalores de $\mathrm{A}^{\mathrm{T}} \mathrm{A}$, causadores da instabilidade e, ao mesmo tempo produzir o menor efeito nos autovalores maiores, associados a parâmetros bem determinados. Assim, qualquer autovalor pequeno vai ser incrementado pela constante " $k$ " e a inversão da matriz $\left(\mathrm{A}^{\mathrm{T}} \mathrm{A}+\right.$ + k I) será mais estável. Bons resultados têm sido obtidos usando a técnica de Marquardt (1970) com um valor de k elevado (aproximadamente 1) quando o valor inicial está longe da solução, o que se constitui no método de gradientes. Um pequeno valor de $\mathrm{k}$ (valores igual ou menores do que 0.01 ) é equivalente para incluir autovalores baixos no estimador e é utilizado quando o estimador está próximo do mínimo, 0 que se constitui na técnica de Newton-Raphson. Incrementar o valor de ' $\mathrm{k}$ ' é similar a descartar os pequenos autovalores e os autovetores associados a ele. Cada autovetor é uma combinação linear dos parâmetros originais do modelo (resistividades e espessuras). (INMAN et al, 1973).

\section{RESULTADOS E INTERPRETAÇÃO}

Na Figura 3 apresentamos os dados do perfil de indução ILD para o poço SA-01, juntamente com o modelo inicial e o perfil litológico obtido a partir da descrição geológica das amostras de calha. Para meIhor análise das respostas obtidas e evitar divergência do algoritmo no processo de inversão, o perfil foi dividido em quatro seções ( $A, B, C$, e D), de modo a individualizar os pacotes de arenito e conglomerado, que possuem maior resistividade. 0 trecho perfilado é caracterizado litologicamente por espessas camadas de folhelhos, chegando a atingir até $50 \mathrm{~m}$ na profundidade de $60-110 \mathrm{~m}$, com uma resistividade média em torno de 6 ohm-m, intercalados por camadas de arenito e conglomerados de menores espessuras. 0 zoneamento do modelo inicial, também apresentado na Figura 4, foi elaborado com o auxílio dos perfis de raios gama e indução, além do estudo das amostras de calhas e dos testemunhos de poço, 0 que permitiu definir melhor as espessuras das camadas. As magnitudes das resistividades das camadas desse modelo foram escolhidas considerando o valor médio de cada zona (TOMASO, 2003).

A Seção A do perfil é apresentada na Figura 4, para a profundidade de 200 - 250m. Esses gráficos mostram o perfil de indução, 0 modelo final resultante do processo de inversão (linha retangular), assim como os valores teóricos equivalentes ao modelo que converge. Nessa seção observa-se uma baixa resolução para as camadas mais finas nesse intervalo, como aparece na profundidade de $230 \mathrm{~m}$ aproximadamente. Na profundidade de $215 \mathrm{~m}$ tem-se alta resistividade devido à presença de uma camada conglomerática bem compactada de $1 \mathrm{~m}$ de espessura.

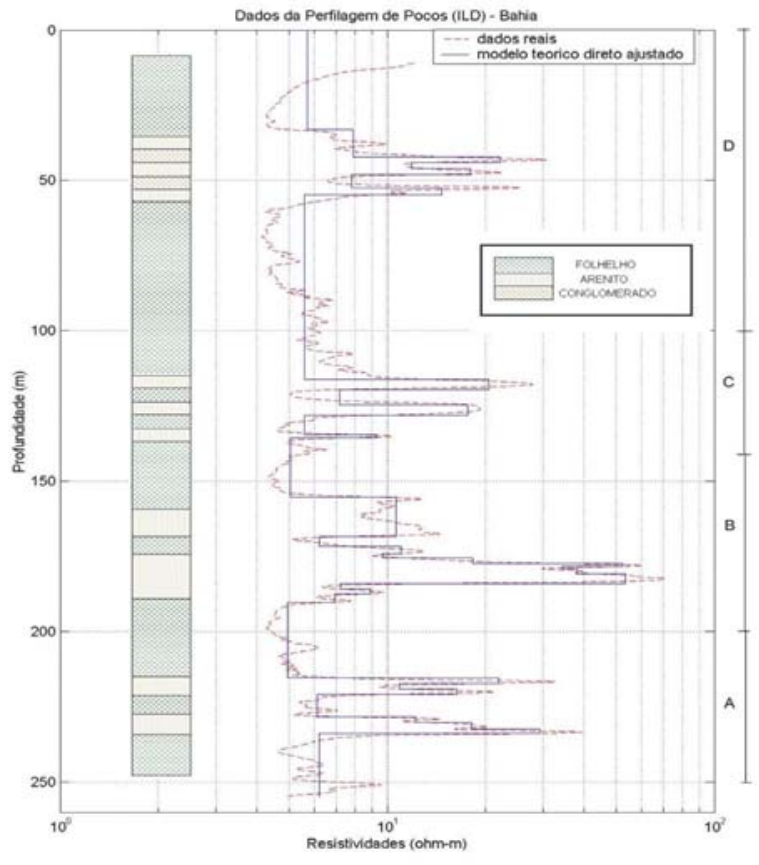

Figura 3 - Perfil Geológico, Dados da Perfilagem e Modelo Inicial - Bahia (TOMASO, 2003). Figure 3 - Geologic register, log data and initial model - Bahia (TOMASO, 2003)

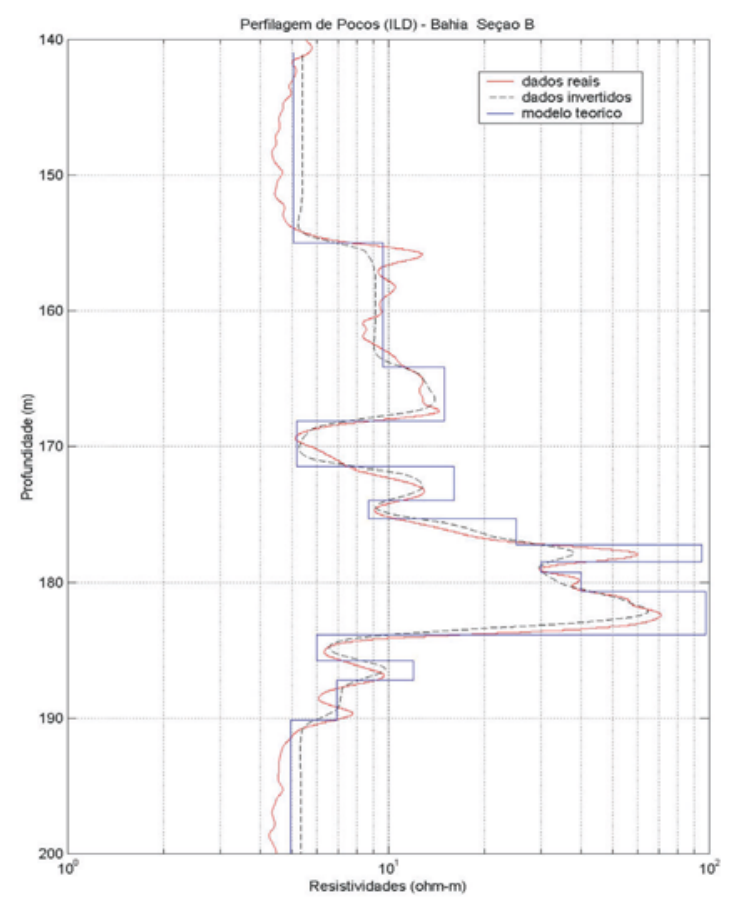

Figura 4 - Inversão do Perfil de Indução para a profundidade de 200-250 metros (Seção A).

Figure 4 - Well logging inversion at 200-250 m (Section A) 
Tabela 1 - Interpretação da Seção A. Table 1 - Section A interpretation.

\begin{tabular}{|c|c|c|c|c|}
\hline \multicolumn{2}{|c|}{ Camadas } & Modelo Inicial & Modelo Final & Diferença \\
\hline \multirow{9}{*}{ 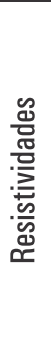 } & 1 & 5,00 & 4,97 & $-0,01$ \\
\hline & 2 & 40,00 & 93,10 & 0,57 \\
\hline & 3 & 10,00 & 9,2 & $.0,09$ \\
\hline & 4 & 16,00 & 33,20 & 0,52 \\
\hline & 5 & 6,00 & 6,10 & 0,02 \\
\hline & 6 & 12,00 & 15,10 & 0,21 \\
\hline & 7 & 18,00 & 20,10 & 0,10 \\
\hline & 8 & 50,00 & 75,30 & 0,34 \\
\hline & 9 & 6,00 & 6,25 & 0,04 \\
\hline \multirow{9}{*}{ 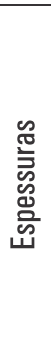 } & 1 & 13,00 & 13,41 & 0,03 \\
\hline & 2 & 2,00 & 2,40 & 0,17 \\
\hline & 3 & 2,00 & 1,52 & $-0,32$ \\
\hline & 4 & 2,00 & 1,92 & $-0,04$ \\
\hline & 5 & 7,00 & 7,36 & 0,05 \\
\hline & 6 & 2,00 & 1,89 & $-0,06$ \\
\hline & 7 & 2,00 & 2,2 & 0,09 \\
\hline & 8 & 2,00 & 2,20 & 0,09 \\
\hline & 9 & 21,00 & 20,30 & $-0,03$ \\
\hline
\end{tabular}

No trecho entre 232,5 - 235m, observa-se também alta resistividade por causa da presença de intercalações de conglomerado e arenito. 0 erro entre os dados experimentais do poço e os do modelo teórico foi de 23\%, o que representa um ajuste razoável. Por outro lado, a Tabela 1 apresenta os resultados da inversão da Seção A com os valores de resistividades e espessuras, tanto do modelo inicial quanto do modelo final. Uma questão relevante apresentada nessa tabela: 0 erro é menor quando consideramos os valores das espessuras, porque se utilizaram valores a priori derivados da interpretaçãa geológica, que são mais confiáveis no processo de inversão.

Na Figura 5, a seção B corresponde à profundidade de 140 até 200 metros. Nessa seçũo, há também perda de resolução para as camadas mais finas quando representamos o perfil sintético. Este efeito é esperado em decorrência do grande contraste produzido pelas camadas adjacentes na profundidade de 155 e 165m, correspondendo a intercalações de arenito, folhelho e conglomerados em níveis milimetricos a centimetricos, adjacente a um pacote de arenito aos $168 \mathrm{~m}$.

Na profundidade 175-185m, observa-se os maiores valores na resistividade, devido à presença de um espesso pacote de rochas conglomeráticas com seixos e grânulos de quartzo, quartzito e rochas gnáissicas. 0 erro de ajuste entre os dados experimentais do poço e os do modelo teórico foi de $16 \%, 0$ que representa um bom ajuste. A Tabela 2 mostra um resumo do processo de inversão da Seção B, com os valores de resistividades e espessuras, tanto do modelo inicial quanto do modelo final. Nesse caso, também e válida a informaç̃ão das espessuras apresentadas para a Seção $\mathrm{A}$.

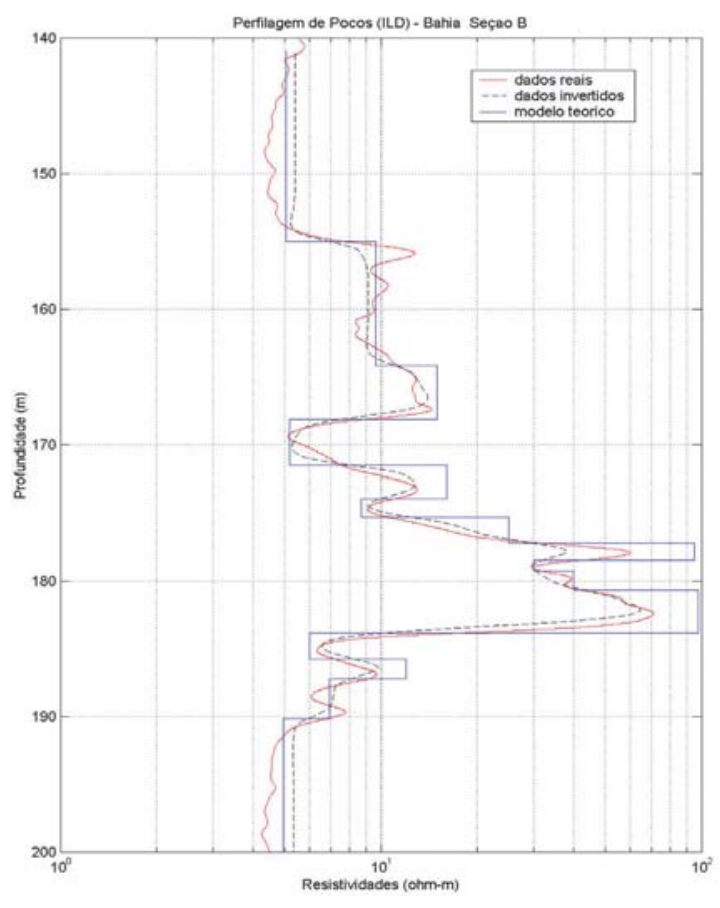

Figura 5 - Inversão do Perfil de Indução para a profundidade de 140-200 metros (Seção B).

Figure 5 - Well logging inversion at 140-200m (Section B).

A Seção C (Figura 6) corresponde à profundidade de 100 até 140 metros. No gráfico nota-se (como no caso anterior), um bom ajuste entre os dados originais do perfil e os dados teóricos. Nesta seção é observado um alto contraste de resistividades entre as camadas, 0 que ocorre devido à intercalação de arenito e folhelho. 0 erro entre os dados experimentais do poço e os do modelo teórico foi de 5\%, 0 que representa um excelente ajuste, apesar de existir a possibilidade de estar modelando ruído, por apresentar um valor menor que 10\%. Erros nos dados podem vir de diferentes fontes: precisão limitada da instrumentação, efeito lateral das heterogeneidades, ruído telúrico e erro no espaçamento para cada intervalo. A Tabela 3 apresenta um resumo da Seçãa $C$ com os valores de resistividades e espessuras, tanto do modelo inicial quanto do modelo final. Observa-se, o mesmo tipo de informação das espessuras mostrada para as seções A e B. 
Tabela 2 - Interpretação da Seção B.

Table 2 - Section B Interpretation.

\begin{tabular}{|c|c|c|c|c|}
\hline \multicolumn{2}{|c|}{ Camadas } & Modelo Inicial & Modelo Final & Diferenca \\
\hline \multirow{15}{*}{ 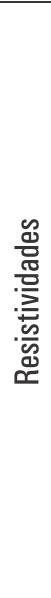 } & 1 & 5,00 & 5,05 & 0,01 \\
\hline & 2 & 10,00 & 9,65 & $-0,04$ \\
\hline & 3 & 10,00 & 15,10 & 0,34 \\
\hline & 4 & 6,00 & 5,19 & $-0,16$ \\
\hline & 5 & 11,00 & 16,04 & 0,31 \\
\hline & 6 & 9,00 & 8,67 & $-0,04$ \\
\hline & 7 & 18,00 & 25,23 & 0,29 \\
\hline & 8 & 52,00 & 95,90 & 0,46 \\
\hline & 9 & 34,00 & 30,20 & $-0,13$ \\
\hline & 10 & 38,00 & 40,20 & 0,05 \\
\hline & 11 & 53,00 & 98,10 & 0,46 \\
\hline & 12 & 7,00 & 6,20 & $-0,13$ \\
\hline & 13 & 9,00 & 12,30 & 0,27 \\
\hline & 14 & 7,00 & 6,91 & $-0,01$ \\
\hline & 15 & 5,00 & 4,97 & $-0,01$ \\
\hline \multirow{15}{*}{ 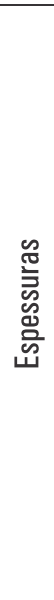 } & 1 & 14,00 & 14,20 & 0,01 \\
\hline & 2 & 9,00 & 9,17 & 0,02 \\
\hline & 3 & 4,00 & 4,40 & 0,09 \\
\hline & 4 & 3,00 & 3,35 & 0,10 \\
\hline & 5 & 2,00 & 2,43 & 0,18 \\
\hline & 6 & 1,00 & 1,36 & 0,26 \\
\hline & 7 & 2,00 & 1,89 & $-0,06$ \\
\hline & 8 & 1,00 & 1,26 & 0,21 \\
\hline & 9 & 1,00 & 0,83 & $-0,20$ \\
\hline & 10 & 1,50 & 1,39 & $-0,08$ \\
\hline & 11 & 3,00 & 3,19 & 0,06 \\
\hline & 12 & 2,00 & 1,89 & $-0,06$ \\
\hline & 13 & 1,50 & 1,44 & $-0,04$ \\
\hline & 14 & 3,00 & 2,96 & $-0,01$ \\
\hline & 15 & 13,00 & 11,73 & $-0,11$ \\
\hline
\end{tabular}

Finalmente para a Seção D do perfil, consideramos a profundidade de 10 até $100 \mathrm{~m}$ (Figura 7). Nessa figura, pode ser observado um bom ajuste entre os perfis real e teórico, para as camadas de conglomerado. Essa seção é caracterizada geologicamente por três níveis de conglomerado com matriz silto-arenosa, cor cinza claro e presença de seixos de quartzo e pelitos, com intercalações subordinadas de arenitos grosseiros. 0 erro entre os dados experimentais do poço e os do modelo teórico foi de 28\%, 0 que representa um ajuste apenas razoável. A Tabela 4 mostra 0 resultado da inversão da Seção D com os valores de resistividades e espessuras, tanto do modelo inicial quanto do modelo final. Novamente, nota-se uma melhor definição das espessuras, como foi discutido anteriormente para as seções $A, B$ e $C$.

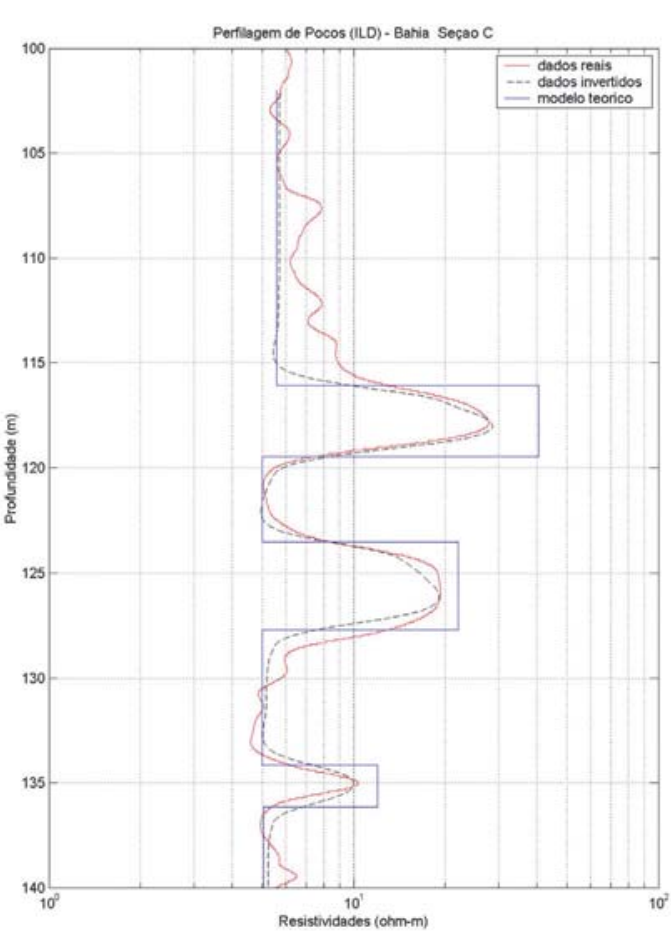

Figura 6 - Inversão do perfil de indução para a profundidade de 100-140m (Seção C).

Figure 6 - Well logging inversion at 100-140m (Section C)

Tabela 3 - Interpretação da Seção C. Table 3 - Section C Interpretation.

\begin{tabular}{|c|c|c|c|c|}
\hline \multicolumn{2}{|c|}{ Camadas } & Modelo Inicial & Modelo Final & Diferença \\
\hline \multirow{7}{*}{ 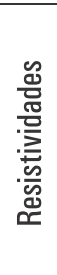 } & 1 & 6,00 & 5,60 & $-0,07$ \\
\hline & 2 & 30,00 & 40,49 & 0,26 \\
\hline & 3 & 7,00 & 5,20 & $.0,35$ \\
\hline & 4 & 17,00 & 22,10 & 0,23 \\
\hline & 5 & 5,00 & 5,20 & 0,04 \\
\hline & 6 & 9,00 & 12,40 & 0,27 \\
\hline & 7 & 6,00 & 5,05 & $.0,19$ \\
\hline \multirow{7}{*}{ 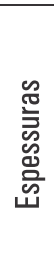 } & 1 & 14,00 & 14,08 & 0,01 \\
\hline & 2 & 3,00 & 3,42 & 0,12 \\
\hline & 3 & 5,00 & 4,30 & $.0,16$ \\
\hline & 4 & 4,00 & 4,20 & 0,05 \\
\hline & 5 & 6,00 & 6,46 & 0,07 \\
\hline & 6 & 1,50 & 2,00 & 0,25 \\
\hline & 7 & 16,00 & 16,46 & 0,03 \\
\hline
\end{tabular}




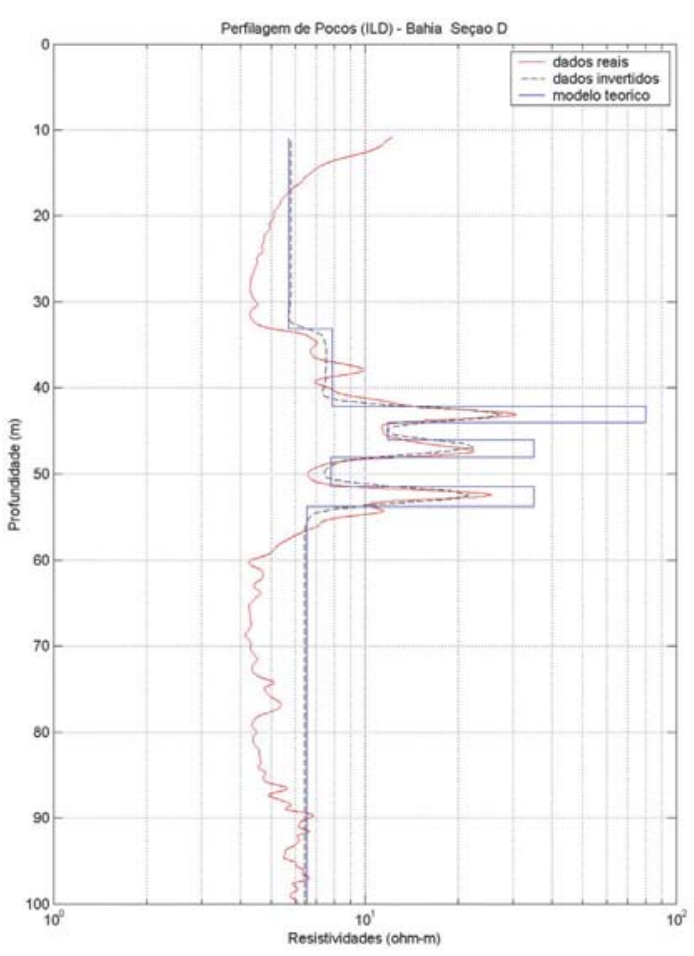

Figura 7 - Inversão do perfil de indução para a profundidade de 0-100m (Seção D).

Figure 7 - Well logging inversion at 0-100m (Section D).

A composição final do perfil, com todas as seções, é mostrada na Figura 8, apresentando perfil de indução, modelo inicial, modelo final de inversão e perfil geológico.

\section{CONCLUSÕES}

Na indústria do petróleo, uma vez perfurado um poço, a realização das perfilagens geofísicas constitui-se num procedimento padrão que fornece uma grande quantidade de parâmetros petrofíisicos. Assim, porosidade, permeabilidade, resistividade, tempo de trânsito, etc., das formações geológicas atravessadas pelo poço, podem ser derivados a partir dos perfis. Nesse sentido, o perfil de indução eletromagnética é importante no conhecimento da resistividade elétrica, podendo indicar a localização das rochas-reservatório e as selantes. Além disso, pode diferenciar os contatos do perfil, 0 que se justifica porque sem ela 0 algoritmo de inversão facilmente diverge devido ao grande número de camadas e inflexões nos dados reais, causado pela riqueza de informações fornecidas pelo perfil. Por outro lado, a tentativa inicial foi realiza-

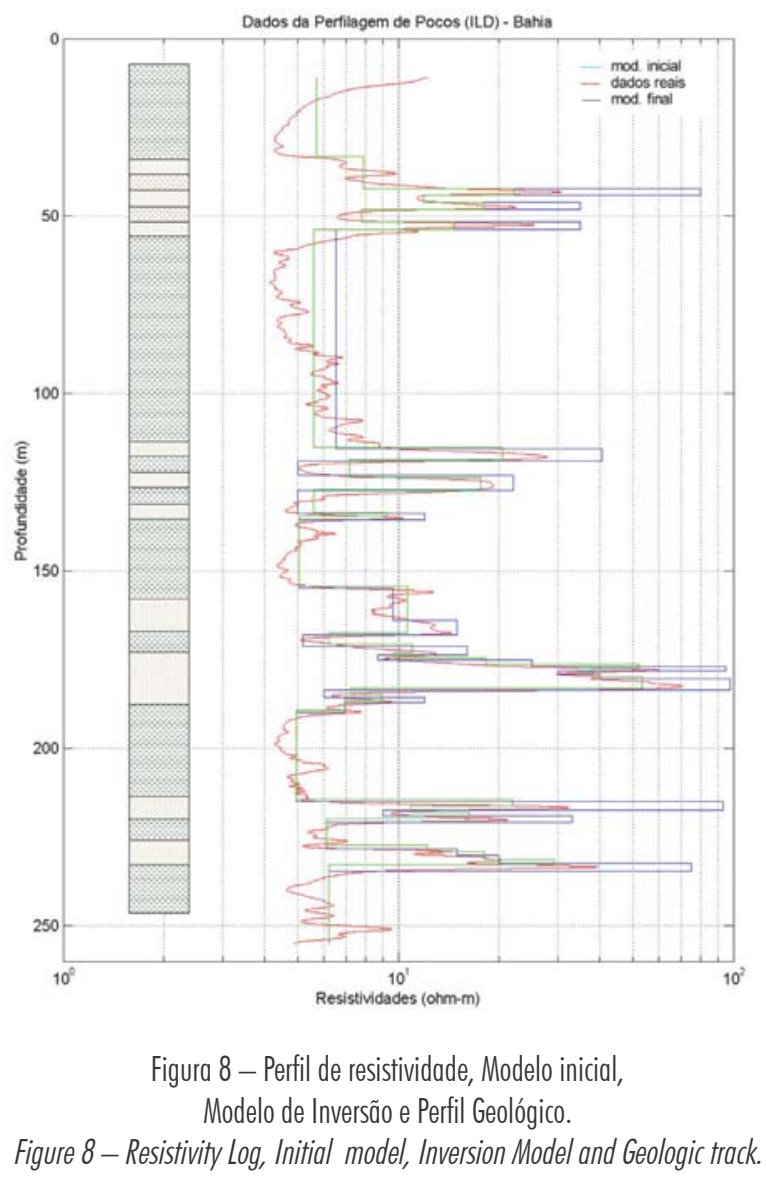

da com base no perfil de indução, considerando a média dos valores para cada camada. Com tal objetivo, também foram utilizados os perfis de raios gama, testemunhos geológicos de poço e amostras de calha. Finalmente, podemos afirmar que os resultados da aplicação da metodologia inversa neste trabalho são bastante satisfatórios, como método auxiliar para caracterização geofísica dos perfis indutivos de poço, principalmente no cálculo das resistividade das camadas. Afirmamos isto porque o perfil teórico resultante da inversão ajusta de maneira satisfatória os dados experimentais do perfil, com erros de ajuste bastante aceitáveis.

\section{REFERÊNCIAS}

BRUHN, C.; MORAES, M. Turbiditos da formação urucutuca na Bacia de Almada, Bahia: um laboratório de campo para estudo de reservatórios canalizados. Boletim de Geociências da Petrobras, [S.I.], v. 3, n. 3, p. 235-267, 1989. 
CaRRASCO, A. M. V.; CARRASQUILLA, A. A. G. Simulação numérica unidimensional de perfis indutivos em poço no caso de reservatórios finamente laminados. Revista Brasileira de Geofísica, v. 21, n. 1. p. 5-12, 2003.

D’AVILA, R. Comunicação pessoal, 2003.

INMAN, J. R. Resistivity inversion with ridge regression. Geophysics, [S.I.], n. 40, p. 798-817, 1975.

INMAN, J. R.; RYU, J.; WARD, S. H. Resistivity inversion (inversão em resistividades). Geophysics, [S.I.], v. 38, p. 1088-1108, 1973.

MARQUARDT, D. W. Generalized inverses, ridge regression, biased linear estimation and nonlinear estimation. Technometrics, n. 12, p. 591-612, 1970.

MENDES, M. P. Evolução, Análise estratigráfica e sistemas turbidíficos em paleocânions submarinos: exemplor de Almada (BA) e Regência (ES). 1998. Dissertação (Mestrado)-Universidade Federal do Rio Grande do Sul, Porto Alegre, 1998.
NERY, G. G.; FEITOSA, F; MANOEL FILHO, J. Hidrogeologia, conceitos e aplicações: perfilagem geofísica aplicada à água subterrânea. Fortaleza: CPRM, 1977.

PELTON, W. H.; RIJO, L.; SWIFT JR, C. M. Inversion of two-dimensional resistivity and induced: polarization data. Geophysics, [S.I.], v. 43, p. 788-803, 1997.

PETRICK, W. R.; PELTON W. H.; WARD, S. H. Ridge regression inversion applied to crustal resistivity sounding data from South Africa. Geophysics, [S.I.], v. 42, p. 995-1005, 1977.

TOMASO K. Relatório técnico preliminar da etapa de perfilagem de polo do projeto turbiditos da Bacia de Almada. Ilhéus: [s.n.], 2003.

WU, F. T. The inverse problem of magnetotelluric sounding. Geophysics, [S.I.], v. 33, p. 972-979, 1968.

\section{NOTAS SOBRE OS AUTORES}

Alfredo M. V. Carrasco é doutorando no programa de Pós-Graduação no Laboratório de Engenharia de Petróleo e Exploração de Petróleo (LENEP), da Universidade Estadual do Norte Fluminense (UENF), Macaé - Brasil, onde está aplicando o algoritmo unidimensional desenvolvido para modelos tridimensionais usando a técnica das equações integrais.

Kledson Tomaso é doutorando no programa de Pós-Graduação no Laboratório de Engenharia de Petróleo e Exploração de Petróleo (LENEP), da Universidade Estadual do Norte Fluminense (UENF), Macaé - Brasil. Atualmente esta começando um programa de treinamento na PETROBRAS.

Antonio Abel González Carrasquilla é professor de Geofísica no Laboratório de Engenharia de Petróleo e Exploração de Petróleo (LENEP), da Universidade Estadual do Norte Fluminense (UENF), desde 1993. É pesquisador II B do CNPq e Chefe do LENEP/UENF. 
